\title{
LA-UR-15-26811
}

Approved for public release; distribution is unlimited.

Title: Nuclear Forensics: Soil Content

Author(s): $\quad$ Beebe, Merilyn Amy

Intended for: Report

Issued: 
Disclaimer:

Los Alamos National Laboratory, an affirmative action/equal opportunity employer,is operated by the Los Alamos National Security, LLC for the National NuclearSecurity Administration of the U.S. Department of Energy under contract DE-AC52-06NA25396. By approving this article, the publisher recognizes that the U.S. Government retains nonexclusive, royalty-free license to publish or reproduce the published form of this contribution, or to allow others to do so, for U.S. Government purposes. Los Alamos National Laboratory requests that the publisher identify this article as work performed under the auspices of the U.S. Departmentof Energy. Los Alamos National Laboratory strongly supports academic freedom and a researcher's right to publish; as an institution, however, the Laboratory does not endorse the viewpoint of a publication or guarantee its technical correctness. 


\title{
Nuclear Forensics: Soil Content
}

\author{
M. A. Beebe \\ Summer Internship Research Review \\ C-NR: Nuclear and Radiochemistry \\ Los Alamos National Laboratory
}

August 6, 2015 


\section{Nuclear Forensics: Soil Content}

\section{NUCLEAR HISTORY}

Nuclear chemistry is a relatively new field of atomic study that began in the late 1800's with the discovery of X-rays by Wilhelm Roentgen and the subsequent discovery of natural radiation in 1896 by Henri Becquerel(“Wilhelm Conrad Röntgen - Biographical” \& "Henri Becquerel - Biographical”). Using this new knowledge, Marie and Pierre Curie experimented with radioactivity and discovered two new radioactive elements, polonium and radium ("Marie Curie."). In the early 1900's Ernest Rutherford revolutionized atomic theory by proving the existence of a positively charged nucleus and in 1905, Einstein's theory of relativity signaled the beginning of the Atomic Age ("Albert Einstein," "The Gold Foil Experiment."). Several years later, in 1939, Otto Hahn and Fritz Strassman discovered the concept of nuclear fission, leading to Enrico Fermi’s experiments with nuclear chain reactions.

In 1942, Fermi successfully created a nuclear chain reaction under the Stagg Field at the University of Chicago, and shortly thereafter, the Manhattan Project was created with the mission of building the world's first atomic bomb ("Enrico Fermi - Biographical"). Robert Oppenheimer, Fermi, and several others were sent to remote location in New Mexico to build 'the gadget.' Within a short period of time, the top-secret work at Los Alamos led to the development of a device ready to be tested. On July 16, 1945, Robert Oppenheimer and his team of scientists detonated the first atomic bomb, changing the course of the war and of history ("The Manhattan Project.").

\section{NUCLEAR POWER TODAY}

While nuclear material is most commonly associated with nuclear weapons, there are several other important uses. Nuclear materials can be used to generate power through contained nuclear fission reactions. The heat produced by such reactions is used to convert water into steam, which then powers generators that convert the mechanical energy into electricity ("Nuclear Power Plants."). In spite of extreme precaution in the building and operation of these plants, they can still be very dangerous. Following the incidents at the Chernobyl and Fukushima nuclear power plants, both domestic and international agencies have implemented new safety measures designed to prevent those kind of accidents from happening in the future. The International Atomic Energy Agency also works to ensure that all facilities containing significant quantities of nuclear material are following international regulations ("Atoms for Peace.").

X-ray machines, CAT scans, and many cancer treatments use radioactive materials. Medical radiation is a significant source of man-made radiation which has led to an increase in the average radiation dose a person can receive in their lifetime (Szaflarski). Even so, the use of nuclear material in medicine has led to vast improvements in diagnostic practices and treatment methods for cancer and other such diseases. 


\section{NUCLEAR FORENSICS}

Following the terrorist attacks of September 11, it is clear that a nuclear attack is a very real and serious possibility. Accompanying an increase in nuclear safeguards and surveillance, a new branch of forensic science has been initiated to focus on the prevention of nuclear terrorism.

Nuclear Forensics is a growing field that is concerned with all stages of the process of creating and detonating a nuclear weapon. The main goal is to prevent nuclear attack by locating and securing nuclear material before it can be used in an aggressive manner. This stage of the process is mostly paperwork; laws, regulations, treaties, and declarations made by individual countries or by the UN Security Council. There is some preliminary leg work done in the form of field testing detection equipment and tracking down orphan materials, however none of these have yielded any spectacular or useful results.

In the event of a nuclear attack, the fist step is to analyze the post detonation debris to aid in the identification of the responsible party. This aspect of the nuclear forensics process, while reactionary, is more scientific. A rock sample taken from the detonation site can be dissolved into liquid form and analyzed to determine its chemical composition. The chemical analysis of spent nuclear material can provide valuable information if properly processed and analyzed.

In order to accurately evaluate the results, scientists require information on the natural occurring elements in the detonation zone. From this information, scientists can determine what percentage of the element originated the bomb itself rather than the environment.

\section{DATA: SOIL CONCENTRATIONS}

The following data set (Tables 1 - 3) contains element concentrations from sixty-nine different cities including the US capitals, the twenty most populous cities in the US, Baltimore and Washington D.C. Cities with no data available are not listed. Information has been collected from the U.S Geological Survey using an interactive map. Links to the specific data locations are listed at the end of this paper. 
1. Data from National Geochemical Survey

\begin{tabular}{|c|c|c|c|c|c|c|}
\hline City & Uranium & Silicon & Iron & Thorium & Strontium & Barium \\
\hline Albuquerque, NM & $<100 \mathrm{ppm}$ & N/A & $2.55 \%$ & $11 \mathrm{ppm}$ & $232 \mathrm{ppm}$ & $662 \mathrm{ppm}$ \\
\hline Annapolis, MD & $<100 \mathrm{ppm}$ & N/A & $5.57 \%$ & $8 \mathrm{ppm}$ & $55 \mathrm{ppm}$ & $156 \mathrm{ppm}$ \\
\hline Atlanta, GA & $<100 \mathrm{ppm}$ & N/A & $0.49 \%$ & 33 ppm & $72 \mathrm{ppm}$ & $605 \mathrm{ppm}$ \\
\hline Augusta, ME & $<100 \mathrm{ppm}$ & $31.50 \%$ & $2.93 \%$ & $13 \mathrm{ppm}$ & $117 \mathrm{ppm}$ & $416 \mathrm{ppm}$ \\
\hline Baton Rouge, MD & $<100 \mathrm{ppm}$ & N/A & $1.59 \%$ & $9 \mathrm{ppm}$ & $91 \mathrm{ppm}$ & $472 \mathrm{ppm}$ \\
\hline Bismarck, ND & $<100 \mathrm{ppm}$ & N/A & $2.55 \%$ & $14 \mathrm{ppm}$ & $78 \mathrm{ppm}$ & $565 \mathrm{ppm}$ \\
\hline Boise, ID & $<100 \mathrm{ppm}$ & N/A & $1.70 \%$ & $19 \mathrm{ppm}$ & $390 \mathrm{ppm}$ & $1030 \mathrm{ppm}$ \\
\hline Carson City, NV & $<100 \mathrm{ppm}$ & N/A & $0.76 \%$ & $<6 \mathrm{ppm}$ & $397 \mathrm{ppm}$ & $1240 \mathrm{ppm}$ \\
\hline Charleston, WV & $<100 \mathrm{ppm}$ & N/A & $2.61 \%$ & $14 \mathrm{ppm}$ & $76 \mathrm{ppm}$ & $521 \mathrm{ppm}$ \\
\hline Cheyenne, WY & $<100 \mathrm{ppm}$ & N/A & $2.57 \%$ & $14 \mathrm{ppm}$ & $164 \mathrm{ppm}$ & $710 \mathrm{ppm}$ \\
\hline Chicago, IL & $<100 \mathrm{ppm}$ & N/A & $2.47 \%$ & $6 \mathrm{ppm}$ & $140 \mathrm{ppm}$ & $550 \mathrm{ppm}$ \\
\hline Columbus, $\mathrm{OH}$ & $<100 \mathrm{ppm}$ & N/A & $2.72 \%$ & $11 \mathrm{ppm}$ & $97 \mathrm{ppm}$ & 519 ppm \\
\hline Concord, NH & $<100 \mathrm{ppm}$ & $33.88 \%$ & $1.61 \%$ & $26 \mathrm{ppm}$ & $168 \mathrm{ppm}$ & $342 \mathrm{ppm}$ \\
\hline Dallas, TX & $<100 \mathrm{ppm}$ & $\mathrm{N} / \mathrm{A}$ & $0.96 \%$ & $10 \mathrm{ppm}$ & $27 \mathrm{ppm}$ & $112 \mathrm{ppm}$ \\
\hline Des Moines, IA & $<100 \mathrm{ppm}$ & N/A & $2.00 \%$ & $10 \mathrm{ppm}$ & $163 \mathrm{ppm}$ & $584 \mathrm{ppm}$ \\
\hline Detroit, MI & $<100 \mathrm{ppm}$ & N/A & $1.86 \%$ & $<6 \mathrm{ppm}$ & $201 \mathrm{ppm}$ & $376 \mathrm{ppm}$ \\
\hline Dover, DE & $<100 \mathrm{ppm}$ & N/A & $2.24 \%$ & $11 \mathrm{ppm}$ & $78 \mathrm{ppm}$ & $494 \mathrm{ppm}$ \\
\hline El Paso, TX & $<100 \mathrm{ppm}$ & N/A & $2.90 \%$ & $8 \mathrm{ppm}$ & $430 \mathrm{ppm}$ & $860 \mathrm{ppm}$ \\
\hline Fort Worth, TX & $<100 \mathrm{ppm}$ & N/A & $2.67 \%$ & $10 \mathrm{ppm}$ & $241 \mathrm{ppm}$ & $207 \mathrm{ppm}$ \\
\hline Frankfort, KY & $<100 \mathrm{ppm}$ & N/A & $4.24 \%$ & $9 \mathrm{ppm}$ & $134 \mathrm{ppm}$ & $317 \mathrm{ppm}$ \\
\hline Hartford, CT & $<100 \mathrm{ppm}$ & $32.97 \%$ & $2.71 \%$ & $15 \mathrm{ppm}$ & $110 \mathrm{ppm}$ & $272 \mathrm{ppm}$ \\
\hline Houston, TX & $<100 \mathrm{ppm}$ & N/A & $0.48 \%$ & $<6 \mathrm{ppm}$ & $10 \mathrm{ppm}$ & $195 \mathrm{ppm}$ \\
\hline Indianapolis, IN & $<100 \mathrm{ppm}$ & N/A & $1.88 \%$ & $<6 \mathrm{ppm}$ & $201 \mathrm{ppm}$ & $251 \mathrm{ppm}$ \\
\hline Jackson, MS & $<100 \mathrm{ppm}$ & $78.20 \%$ & $2.60 \%$ & $12 \mathrm{ppm}$ & 73 ppm & $536 \mathrm{ppm}$ \\
\hline Jacksonville, FL & $<100 \mathrm{ppm}$ & $92 \%$ & $0.39 \%$ & $8 \mathrm{ppm}$ & $30 \mathrm{ppm}$ & 99 ppm \\
\hline Jefferson City, MO & $<100 \mathrm{ppm}$ & N/A & $1.96 \%$ & $10 \mathrm{ppm}$ & $73 \mathrm{ppm}$ & $635 \mathrm{ppm}$ \\
\hline Juneau, AK & $<100 \mathrm{ppm}$ & N/A & $5.46 \%$ & $14 \mathrm{ppm}$ & $170 \mathrm{ppm}$ & $2370 \mathrm{ppm}$ \\
\hline Lincoln, NE & $<100 \mathrm{ppm}$ & N/A & $2.32 \%$ & $10 \mathrm{ppm}$ & $149 \mathrm{ppm}$ & $786 \mathrm{ppm}$ \\
\hline Little Rock, AR & $<100 \mathrm{ppm}$ & N/A & $2.58 \%$ & $12 \mathrm{ppm}$ & $58 \mathrm{ppm}$ & $304 \mathrm{ppm}$ \\
\hline Los Angeles, CA & $<100 \mathrm{ppm}$ & N/A & $3.50 \%$ & $15 \mathrm{ppm}$ & $499 \mathrm{ppm}$ & $888 \mathrm{ppm}$ \\
\hline Montpelier, VT & $<100 \mathrm{ppm}$ & $28.21 \%$ & $5.71 \%$ & $10 \mathrm{ppm}$ & $97 \mathrm{ppm}$ & $117 \mathrm{ppm}$ \\
\hline Nashville, TN & $<100 \mathrm{ppm}$ & N/A & $3.99 \%$ & $12 \mathrm{ppm}$ & $187 \mathrm{ppm}$ & $424 \mathrm{ppm}$ \\
\hline Oklahoma City, OK & $<100 \mathrm{ppm}$ & N/A & $1.60 \%$ & $8 \mathrm{ppm}$ & $68 \mathrm{ppm}$ & 769 ppm \\
\hline Phoenix, AZ & $<100 \mathrm{ppm}$ & $\mathrm{N} / \mathrm{A}$ & $4.17 \%$ & $12 \mathrm{ppm}$ & $336 \mathrm{ppm}$ & $610 \mathrm{ppm}$ \\
\hline Pierre, SD & $<100 \mathrm{ppm}$ & N/A & $2.03 \%$ & $8 \mathrm{ppm}$ & $151 \mathrm{ppm}$ & $780 \mathrm{ppm}$ \\
\hline Raleigh, NC & $<100 \mathrm{ppm}$ & N/A & $3.10 \%$ & $14 \mathrm{ppm}$ & 193 ppm & $644 \mathrm{ppm}$ \\
\hline Richmond, VA & $<100 \mathrm{ppm}$ & N/A & $2.96 \%$ & $13 \mathrm{ppm}$ & $27 \mathrm{ppm}$ & $232 \mathrm{ppm}$ \\
\hline Sacramento, CA & $<100 \mathrm{ppm}$ & N/A & $2.77 \%$ & $6 \mathrm{ppm}$ & $214 \mathrm{ppm}$ & $553 \mathrm{ppm}$ \\
\hline Salem, OR & $<100 \mathrm{ppm}$ & N/A & $7.68 \%$ & $12 \mathrm{ppm}$ & $133 \mathrm{ppm}$ & $585 \mathrm{ppm}$ \\
\hline Salt Lake City, UT & $<100 \mathrm{ppm}$ & N/A & $1.92 \%$ & $11 \mathrm{ppm}$ & 99 ppm & $366 \mathrm{ppm}$ \\
\hline San Antonio, TX & $<100 \mathrm{ppm}$ & N/A & $2.15 \%$ & $<6 \mathrm{ppm}$ & $192 \mathrm{ppm}$ & $190 \mathrm{ppm}$ \\
\hline San Jose, CA & $<100 \mathrm{ppm}$ & N/A & $3.51 \%$ & $9 \mathrm{ppm}$ & $1443 \mathrm{ppm}$ & $585 \mathrm{ppm}$ \\
\hline Santa Fe, NM & $<100 \mathrm{ppm}$ & N/A & $5.83 \%$ & $20 \mathrm{ppm}$ & $198 \mathrm{ppm}$ & $782 \mathrm{ppm}$ \\
\hline Springfield, IL & $<100 \mathrm{ppm}$ & N/A & $2.57 \%$ & $12 \mathrm{ppm}$ & $107 \mathrm{ppm}$ & $641 \mathrm{ppm}$ \\
\hline Tallahassee, FL & $<100 \mathrm{ppm}$ & N/A & $1.14 \%$ & $10 \mathrm{ppm}$ & $98 \mathrm{ppm}$ & $230 \mathrm{ppm}$ \\
\hline
\end{tabular}




\begin{tabular}{|l|c|c|c|c|c|c|}
\hline Topeka, KS & $<100 \mathrm{ppm}$ & N/A & $2.20 \%$ & $9 \mathrm{ppm}$ & $112 \mathrm{ppm}$ & $483 \mathrm{ppm}$ \\
\hline
\end{tabular}

\section{Data from National Uranium Resource Evaluation Program}

\begin{tabular}{|c|c|c|c|c|c|}
\hline City & Uranium & Iron & Thorium & Strontium & Barium \\
\hline Albany, NY & $0.5 \mathrm{ppm}$ & $2.73 \%$ & $5 \mathrm{ppm}$ & N/A & $212 \mathrm{ppm}$ \\
\hline Annapolis, MD & $12.1 \mathrm{ppm}$ & $5.00 \%$ & $58 \mathrm{ppm}$ & N/A & $\mathrm{N} / \mathrm{A}$ \\
\hline Atlanta, GA & $7.5 \mathrm{ppm}$ & $3.28 \%$ & $24 \mathrm{ppm}$ & N/A & $128 \mathrm{ppm}$ \\
\hline Augusta, ME & $2.3 \mathrm{ppm}$ & $2.64 \%$ & $6 \mathrm{ppm}$ & $102 \mathrm{ppm}$ & $348 \mathrm{ppm}$ \\
\hline Austin, TX & $2.5 \mathrm{ppm}$ & $1.88 \%$ & $10 \mathrm{ppm}$ & $145 \mathrm{ppm}$ & $291 \mathrm{ppm}$ \\
\hline Boise, ID & $3.3 \mathrm{ppm}$ & $2.29 \%$ & $12 \mathrm{ppm}$ & $350 \mathrm{ppm}$ & 808 ppm \\
\hline Carson City, NV & $1.9 \mathrm{ppm}$ & $2.30 \%$ & $6 \mathrm{ppm}$ & $60 \mathrm{ppm}$ & $382 \mathrm{ppm}$ \\
\hline Cheyenne, WY & $3 \mathrm{ppm}$ & $1.93 \%$ & $8 \mathrm{ppm}$ & $172 \mathrm{ppm}$ & $607 \mathrm{ppm}$ \\
\hline Columbia, SC & $25.1 \mathrm{ppm}$ & $2.71 \%$ & $120 \mathrm{ppm}$ & $<50 \mathrm{ppm}$ & N/A \\
\hline Concord, NH & $4.6 \mathrm{ppm}$ & $2.71 \%$ & $23 \mathrm{ppm}$ & $53 \mathrm{ppm}$ & $123 \mathrm{ppm}$ \\
\hline Dallas, TX & $1.7 \mathrm{ppm}$ & $1.35 \%$ & $<2 \mathrm{ppm}$ & $731 \mathrm{ppm}$ & $177 \mathrm{ppm}$ \\
\hline Denver, CO & $5 \mathrm{ppm}$ & $8.10 \%$ & $18.8 \mathrm{ppm}$ & $670 \mathrm{ppm}$ & $677 \mathrm{ppm}$ \\
\hline Dover, DE & $8.1 \mathrm{ppm}$ & $9.57 \%$ & $35 \mathrm{ppm}$ & N/A & N/A \\
\hline Fort Worth, TX & $1.5 \mathrm{ppm}$ & $1.51 \%$ & $6 \mathrm{ppm}$ & $400 \mathrm{ppm}$ & $143 \mathrm{ppm}$ \\
\hline Harrisburg, PA & $0.3 \mathrm{ppm}$ & $2.92 \%$ & $8 \mathrm{ppm}$ & N/A & $227 \mathrm{ppm}$ \\
\hline Hartford, CT & $9.9 \mathrm{ppm}$ & $7.03 \%$ & $32 \mathrm{ppm}$ & N/A & N/A \\
\hline Helena, MT & $3.1 \mathrm{ppm}$ & $1.33 \%$ & $10.6 \mathrm{ppm}$ & $<321 \mathrm{ppm}$ & $705 \mathrm{ppm}$ \\
\hline Juneau, AK & $1.35 \mathrm{ppm}$ & $8.98 \%$ & $<1.9 \mathrm{ppm}$ & $<411 \mathrm{ppm}$ & $<207 \mathrm{ppm}$ \\
\hline Lincoln, NE & $3 \mathrm{ppm}$ & $2.39 \%$ & $8 \mathrm{ppm}$ & $178 \mathrm{ppm}$ & $827 \mathrm{ppm}$ \\
\hline Little Rock, AR & $2.2 \mathrm{ppm}$ & $3.15 \%$ & $10 \mathrm{ppm}$ & N/A & $55.3 \%$ \\
\hline Montgomery, AL & $55.3 \mathrm{ppm}$ & $4.14 \%$ & $357 \mathrm{ppm}$ & N/A & N/A \\
\hline Montpelier, VT & $2 \mathrm{ppm}$ & $<0.93 \%$ & $5 \mathrm{ppm}$ & N/A & N/A \\
\hline $\begin{array}{l}\text { Oklahoma City, } \\
\text { OK }\end{array}$ & $1.84 \%$ & $0.72 \%$ & $6 \mathrm{ppm}$ & $79 \mathrm{ppm}$ & $1613 \mathrm{ppm}$ \\
\hline Phoenix, AZ & $2.5 \mathrm{ppm}$ & $4.62 \%$ & $17 \mathrm{ppm}$ & N/A & N/A \\
\hline Providence, RI & $2.8 \mathrm{ppm}$ & $1.57 \%$ & $5 \mathrm{ppm}$ & $\mathrm{N} / \mathrm{A}$ & N/A \\
\hline Raleigh, NC & $13.9 \mathrm{ppm}$ & $2.11 \%$ & $2 \mathrm{ppm}$ & N/A & $32 \mathrm{ppm}$ \\
\hline Richmond, VA & $8.7 \mathrm{ppm}$ & $4.49 \%$ & $27 \mathrm{ppm}$ & $\mathrm{N} / \mathrm{A}$ & N/A \\
\hline Sacramento, CA & $2.8 \mathrm{ppm}$ & $3.46 \%$ & $4 \mathrm{ppm}$ & $196 \mathrm{ppm}$ & $511 \mathrm{ppm}$ \\
\hline Salt Lake City, UT & $4.2 \mathrm{ppm}$ & $2.53 \%$ & $11 \mathrm{ppm}$ & $153 \mathrm{ppm}$ & $964 \mathrm{ppm}$ \\
\hline San Antonio, TX & $2.03 \mathrm{ppm}$ & N/A & $<400 \mathrm{ppm}$ & $\mathrm{N} / \mathrm{A}$ & $100 \mathrm{ppm}$ \\
\hline Santa Fe, NM & $4.77 \mathrm{ppm}$ & $4.52 \%$ & $17.5 \mathrm{ppm}$ & $<252 \mathrm{ppm}$ & $468 \mathrm{ppm}$ \\
\hline Trenton, NJ & $0.8 \mathrm{ppm}$ & $3.90 \%$ & $25 \mathrm{ppm}$ & N/A & $107 \mathrm{~m}$ \\
\hline
\end{tabular}


Data from Rock Analysis

\begin{tabular}{|c|c|c|c|c|c|c|c|c|c|c|c|}
\hline City & $\overline{\mathrm{U}}$ & Si & $\mathrm{SiO} 2$ & $\mathrm{CaO}$ & $\mathrm{K} 20$ & $\begin{array}{l}\mathrm{Na} 2 \\
\mathrm{O}\end{array}$ & Al2O3 & Fe & Th & $\mathrm{Sr}$ & $\mathbf{B a}$ \\
\hline Albuquerque, NM & $\begin{array}{c}\text { N0 } \\
\text { ppm }\end{array}$ & $\begin{array}{c}\text { G10 } \\
\%\end{array}$ & $\begin{array}{c}50.90 \\
\%\end{array}$ & $\begin{array}{c}9.20 \\
\%\end{array}$ & $\begin{array}{c}0.42 \\
\%\end{array}$ & $\begin{array}{c}2.39 \\
\%\end{array}$ & $\begin{array}{l}16 \\
\%\end{array}$ & $\begin{array}{l}7 \\
\% \\
\end{array}$ & $\begin{array}{c}\text { N0 } \\
\text { ppm }\end{array}$ & $\begin{array}{r}700 \\
\text { ppm }\end{array}$ & $\begin{array}{r}150 \\
\mathrm{ppm}\end{array}$ \\
\hline Atlanta, GA & $\begin{array}{l}0.19 \\
\mathrm{ppm}\end{array}$ & $\begin{array}{l}27 \\
\%\end{array}$ & $\begin{array}{c}58.30 \\
\%\end{array}$ & $\begin{array}{c}0.06 \\
\%\end{array}$ & $\begin{array}{c}\text { L0.02 } \\
\%\end{array}$ & $\begin{array}{c}\text { L0.15 } \\
\%\end{array}$ & $\begin{array}{c}0.91 \\
\%\end{array}$ & $\begin{array}{c}5.49 \\
\%\end{array}$ & $\begin{array}{c}0.5 \\
\mathrm{ppm}\end{array}$ & $\begin{array}{l}\text { L15 } \\
\text { ppm }\end{array}$ & $\begin{array}{l}\text { L90 } \\
\text { ppm }\end{array}$ \\
\hline Augusta, ME & $\begin{array}{c}\text { N0 } \\
\text { ppm }\end{array}$ & N/A & $\begin{array}{c}55.80 \\
\%\end{array}$ & $\begin{array}{c}5.80 \\
\%\end{array}$ & $\begin{array}{c}1.50 \\
\%\end{array}$ & $\begin{array}{c}0.20 \\
\%\end{array}$ & $\begin{array}{l}16 \\
\%\end{array}$ & N/A & $\begin{array}{c}\text { N0 } \\
\text { ppm }\end{array}$ & $\begin{array}{l}500 \\
\mathrm{ppm}\end{array}$ & $\begin{array}{l}150 \\
\text { ppm }\end{array}$ \\
\hline Austin, TX & N/A & N/A & N/A & $\begin{array}{c}0.44 \\
\%\end{array}$ & $\mathrm{~N} / \mathrm{A}$ & N/A & $\mathrm{N} / \mathrm{A}$ & N/A & N/A & N/A & N/A \\
\hline Baltimore, MA & $\begin{array}{c}\text { L0.4p } \\
\text { pm }\end{array}$ & N/A & $\begin{array}{c}47.90 \\
\%\end{array}$ & $\begin{array}{c}10.80 \\
\%\end{array}$ & $\begin{array}{c}0.38 \\
\%\end{array}$ & $\begin{array}{c}2.56 \\
\%\end{array}$ & $\begin{array}{c}15.40 \\
\%\end{array}$ & $\begin{array}{c}8.86 \\
\%\end{array}$ & $\begin{array}{l}\mathrm{L} 0.2 \\
\mathrm{ppm}\end{array}$ & $\begin{array}{c}89 \\
\mathrm{ppm}\end{array}$ & $\begin{array}{c}36 \\
\mathrm{ppm}\end{array}$ \\
\hline Boise, ID & $\mathrm{N} / \mathrm{A}$ & N/A & $\begin{array}{c}72.10 \\
\% \\
\end{array}$ & $\begin{array}{c}2.34 \\
\% \\
\end{array}$ & $\begin{array}{c}2.78 \\
\%\end{array}$ & $\begin{array}{c}4.03 \\
\%\end{array}$ & $\begin{array}{c}15.20 \\
\%\end{array}$ & N/A & N/A & $\begin{array}{l}570 \\
\mathrm{ppm}\end{array}$ & $\begin{array}{l}1390 \\
\text { ppm }\end{array}$ \\
\hline Carson City, NV & $\begin{array}{l}4.25 \\
\mathrm{ppm}\end{array}$ & N/A & N/A & N/A & N/A & N/A & $\mathrm{N} / \mathrm{A}$ & $\begin{array}{c}0.80 \\
\% \\
\end{array}$ & $\begin{array}{l}12.5 \\
\mathrm{ppm}\end{array}$ & $\begin{array}{l}\text { L100 } \\
\text { ppm }\end{array}$ & $\begin{array}{r}644 \\
\mathrm{ppm}\end{array}$ \\
\hline Charleston, WV & $\begin{array}{l}\text { L220 } \\
\text { ppm }\end{array}$ & $\begin{array}{l}28 \\
\%\end{array}$ & $\begin{array}{c}56.40 \\
\%\end{array}$ & $\begin{array}{c}0.45 \\
\%\end{array}$ & $\begin{array}{c}0.16 \\
\%\end{array}$ & $\begin{array}{c}\mathrm{L} 0.15 \\
\%\end{array}$ & $\begin{array}{c}27.50 \\
\%\end{array}$ & $\begin{array}{c}0.64 \\
\%\end{array}$ & $\begin{array}{l}\text { L46 } \\
\text { ppm }\end{array}$ & $\begin{array}{l}350 \\
\text { ppm }\end{array}$ & $\begin{array}{c}90 \\
\mathrm{ppm}\end{array}$ \\
\hline Charlotte, NC & N/A & N/A & $\begin{array}{l}53 \\
\%\end{array}$ & $\begin{array}{c}9.90 \\
\%\end{array}$ & $\begin{array}{c}0.50 \\
\%\end{array}$ & $\begin{array}{c}2.50 \\
\%\end{array}$ & $\begin{array}{c}17.10 \\
\%\end{array}$ & N/A & N/A & N/A & N/A \\
\hline Cheyenne, WY & $\begin{array}{l}7.31 \\
\mathrm{ppm}\end{array}$ & N/A & $\begin{array}{c}73.70 \\
\%\end{array}$ & $\begin{array}{c}0.83 \\
\%\end{array}$ & $\begin{array}{c}5.74 \\
\%\end{array}$ & $\begin{array}{c}1.67 \\
\%\end{array}$ & $\begin{array}{c}11.70 \\
\%\end{array}$ & N/A & $\begin{array}{c}30 \\
\mathrm{ppm}\end{array}$ & $\begin{array}{c}0.01 \\
\%\end{array}$ & N/A \\
\hline Columbia, SC & $\begin{array}{c}\text { N0 } \\
\text { ppm }\end{array}$ & N/A & $\begin{array}{c}72.30 \\
\%\end{array}$ & $\begin{array}{c}1.30 \\
\%\end{array}$ & $\begin{array}{c}0.35 \\
\%\end{array}$ & $\begin{array}{c}5.50 \\
\%\end{array}$ & $\begin{array}{c}11.50 \\
\%\end{array}$ & N/A & $\begin{array}{c}\text { N0 } \\
\text { ppm }\end{array}$ & $\begin{array}{c}70 \\
\mathrm{ppm}\end{array}$ & $\begin{array}{l}150 \\
\mathrm{ppm}\end{array}$ \\
\hline Columbus, $\mathrm{OH}$ & $\begin{array}{c}\text { N0 } \\
\text { ppm }\end{array}$ & $\mathrm{N} / \mathrm{A}$ & $\mathrm{N} / \mathrm{A}$ & $\mathrm{N} / \mathrm{A}$ & N/A & $\mathrm{N} / \mathrm{A}$ & N/A & $\begin{array}{c}1.50 \\
\%\end{array}$ & $\begin{array}{c}\text { N0 } \\
\text { ppm }\end{array}$ & $\begin{array}{l}500 \\
\mathrm{ppm}\end{array}$ & $\begin{array}{l}100 \\
\mathrm{ppm}\end{array}$ \\
\hline Concord, NH & $\begin{array}{l}\mathrm{L} 0.8 \\
\mathrm{ppm}\end{array}$ & N/A & $\begin{array}{c}69.30 \\
\%\end{array}$ & $\begin{array}{c}3.70 \\
\%\end{array}$ & $\begin{array}{c}1.50 \\
\%\end{array}$ & $\begin{array}{c}4 \\
\%\end{array}$ & $\begin{array}{c}16.90 \\
\%\end{array}$ & $\begin{array}{c}2.00 \\
\%\end{array}$ & $\begin{array}{c}6 \\
\mathrm{ppm}\end{array}$ & N/A & $\begin{array}{r}264 \\
\mathrm{ppm}\end{array}$ \\
\hline El Paso, TX & $\begin{array}{c}\text { N0 } \\
\text { ppm }\end{array}$ & $\begin{array}{c}\text { G10 } \\
\%\end{array}$ & $\begin{array}{c}62.50 \\
\%\end{array}$ & $\begin{array}{c}3.60 \\
\%\end{array}$ & $\begin{array}{c}2.40 \\
\%\end{array}$ & $\begin{array}{c}5.60 \\
\%\end{array}$ & $\begin{array}{c}18.30 \\
\%\end{array}$ & $\begin{array}{c}3 \\
\%\end{array}$ & $\begin{array}{c}\text { N0 } \\
\text { ppm }\end{array}$ & $\begin{array}{l}1500 \\
\text { ppm }\end{array}$ & $\begin{array}{l}1500 \\
\mathrm{ppm}\end{array}$ \\
\hline Frankfort, KY & $\begin{array}{l}\mathrm{L} 215 \\
\mathrm{ppm}\end{array}$ & $\begin{array}{c}0.59 \\
\%\end{array}$ & $\begin{array}{c}0.80 \\
\%\end{array}$ & $\begin{array}{c}54.40 \\
\%\end{array}$ & $\begin{array}{c}0.11 \\
\%\end{array}$ & $\begin{array}{c}0.10 \\
\%\end{array}$ & $\begin{array}{c}0.16 \\
\%\end{array}$ & $\begin{array}{c}0.27 \\
\%\end{array}$ & $\begin{array}{c}\text { L21.5 } \\
\text { ppm }\end{array}$ & $\begin{array}{c}\text { G464 } \\
\text { ppm }\end{array}$ & $\begin{array}{l}130 \\
\mathrm{ppm}\end{array}$ \\
\hline Harrisburg, PA & $\begin{array}{l}\mathrm{L} 0.6 \\
\mathrm{ppm}\end{array}$ & $\mathrm{N} / \mathrm{A}$ & $\begin{array}{c}52.10 \\
\%\end{array}$ & $\begin{array}{c}12.10 \\
\% \\
\end{array}$ & $\begin{array}{c}0.42 \\
\% \\
\end{array}$ & $\begin{array}{c}1.84 \\
\%\end{array}$ & $\begin{array}{c}14.70 \\
\%\end{array}$ & $\begin{array}{c}7.03 \\
\%\end{array}$ & $\begin{array}{c}1.5 \\
\mathrm{ppm}\end{array}$ & $\begin{array}{l}199 \\
\mathrm{ppm}\end{array}$ & $\begin{array}{l}122 \\
\mathrm{ppm}\end{array}$ \\
\hline Hartford, CT & $\begin{array}{l}\text { L220 } \\
\text { ppm }\end{array}$ & $\begin{array}{c}\text { G34 } \\
\% \\
\end{array}$ & N/A & $\mathrm{N} / \mathrm{A}$ & N/A & N/A & N/A & $\begin{array}{c}6.20 \\
\% \\
\end{array}$ & $\begin{array}{l}\mathrm{L} 46 \\
\mathrm{ppm}\end{array}$ & $\begin{array}{c}23 \\
\mathrm{ppm}\end{array}$ & $\begin{array}{l}180 \\
\mathrm{ppm}\end{array}$ \\
\hline Honolulu, HI & N/A & N/A & $\begin{array}{l}45 \\
\%\end{array}$ & $\begin{array}{c}2.23 \\
\%\end{array}$ & $\begin{array}{c}\mathrm{L} 0.00 \\
2 \%\end{array}$ & $\begin{array}{c}0.09 \\
\%\end{array}$ & $2.41 \%$ & N/A & N/A & N/A & N/A \\
\hline Jefferson City, MO & $\begin{array}{c}\text { N0 } \\
\text { ppm }\end{array}$ & $\begin{array}{c}\text { G10 } \\
\%\end{array}$ & $\begin{array}{l}41 \\
\%\end{array}$ & $\begin{array}{c}0.20 \\
\%\end{array}$ & $\begin{array}{c}1.30 \\
\%\end{array}$ & $\begin{array}{c}0.08 \\
\%\end{array}$ & $\begin{array}{l}33 \\
\%\end{array}$ & $\begin{array}{c}0.50 \\
\%\end{array}$ & $\begin{array}{c}\text { N0 } \\
\text { ppm }\end{array}$ & $\begin{array}{l}150 \\
\mathrm{ppm}\end{array}$ & $\begin{array}{c}30 \\
\mathrm{ppm}\end{array}$ \\
\hline
\end{tabular}




\begin{tabular}{|c|c|c|c|c|c|c|c|c|c|c|c|}
\hline Juneau, AK & $\begin{array}{l}0.33 \\
\mathrm{ppm}\end{array}$ & N/A & $\begin{array}{c}50.20 \\
\%\end{array}$ & $\begin{array}{c}7.91 \\
\%\end{array}$ & $\begin{array}{c}0.44 \\
\%\end{array}$ & $\begin{array}{c}4.10 \\
\%\end{array}$ & $\begin{array}{l}14 \\
\%\end{array}$ & $\begin{array}{c}7.01 \\
\%\end{array}$ & $\begin{array}{l}0.74 \\
\mathrm{ppm}\end{array}$ & $\begin{array}{l}250 \\
\mathrm{ppm}\end{array}$ & $\begin{array}{c}90 \\
\mathrm{ppm}\end{array}$ \\
\hline Little Rock, AR & $\begin{array}{c}\text { N0 } \\
\text { ppm }\end{array}$ & $\begin{array}{c}\text { G10 } \\
\% \\
\end{array}$ & N/A & N/A & N/A & N/A & N/A & $\begin{array}{c}5.00 \\
\%\end{array}$ & $\begin{array}{c}\text { N0 } \\
\text { ppm }\end{array}$ & $\begin{array}{l}100 \\
\mathrm{ppm}\end{array}$ & $\begin{array}{r}300 \\
\mathrm{ppm} \\
\end{array}$ \\
\hline Los Angeles, CA & $\begin{array}{l}0.73 \\
\mathrm{ppm}\end{array}$ & N/A & N/A & N/A & N/A & N/A & N/A & N/A & $\begin{array}{r}3.5 \\
\mathrm{ppm}\end{array}$ & N/A & N/A \\
\hline Montpelier, VT & $\begin{array}{l}\mathrm{L} 400 \\
\mathrm{ppm} \\
\end{array}$ & N/A & $\begin{array}{c}42.70 \\
\% \\
\end{array}$ & $\begin{array}{c}0.03 \\
\% \\
\end{array}$ & $\begin{array}{c}\mathrm{L} 0.02 \\
\% \\
\end{array}$ & $\begin{array}{c}\text { L0.15 } \\
\% \\
\end{array}$ & $\begin{array}{c}1.11 \\
\%\end{array}$ & $\begin{array}{c}5.60 \\
\% \\
\end{array}$ & $\begin{array}{l}\mathrm{L} 20 \\
\mathrm{ppm} \\
\end{array}$ & $\begin{array}{c}\mathrm{L} 8 \\
\mathrm{ppm} \\
\end{array}$ & $\begin{array}{r}\mathrm{L} 4 \\
\mathrm{ppm}\end{array}$ \\
\hline Nashville, TN & $\begin{array}{l}\text { L100 } \\
\text { ppm }\end{array}$ & N/A & N/A & N/A & $\mathrm{N} / \mathrm{A}$ & N/A & N/A & $\begin{array}{c}5.90 \\
\% \\
\end{array}$ & $\begin{array}{c}\text { L5 } \\
\text { ppm } \\
\end{array}$ & $\begin{array}{c}47 \\
\mathrm{ppm}\end{array}$ & $\begin{array}{l}160 \\
\mathrm{ppm} \\
\end{array}$ \\
\hline New York, NY & $\begin{array}{l}\mathrm{L} 215 \\
\mathrm{ppm}\end{array}$ & $\begin{array}{c}24.30 \\
\% \\
\end{array}$ & $\begin{array}{c}52.40 \\
\%\end{array}$ & $\begin{array}{c}10.70 \\
\%\end{array}$ & $\begin{array}{c}0.64 \\
\%\end{array}$ & $\begin{array}{c}2 \\
\% \\
\end{array}$ & $\begin{array}{l}15 \\
\% \\
\end{array}$ & $\begin{array}{c}10.20 \\
\%\end{array}$ & $\begin{array}{l}\mathrm{L} 21.5 \\
\mathrm{ppm}\end{array}$ & $\begin{array}{r}237 \\
\mathrm{ppm}\end{array}$ & $\begin{array}{l}215 \\
\mathrm{ppm}\end{array}$ \\
\hline $\begin{array}{l}\text { Oklahoma City, } \\
\text { OK }\end{array}$ & $\begin{array}{l}1.15 \\
\mathrm{ppm}\end{array}$ & N/A & N/A & $\mathrm{N} / \mathrm{A}$ & N/A & N/A & N/A & $\begin{array}{c}3.50 \\
\%\end{array}$ & $\begin{array}{c}4.64 \\
\% \\
\end{array}$ & $\begin{array}{c}44 \\
\mathrm{ppm}\end{array}$ & $\begin{array}{c}66 \\
\mathrm{ppm}\end{array}$ \\
\hline Phoenix, AZ & $\begin{array}{l}2.02 \\
\mathrm{ppm}\end{array}$ & N/A & N/A & $\begin{array}{c}7.89 \\
\% \\
\end{array}$ & $\begin{array}{c}1.93 \\
\%\end{array}$ & $\begin{array}{c}3.59 \\
\%\end{array}$ & $\begin{array}{c}14.70 \\
\% \\
\end{array}$ & $\begin{array}{c}6.51 \\
\% \\
\end{array}$ & $\begin{array}{c}6.18 \\
\%\end{array}$ & $\begin{array}{l}1000 \\
\mathrm{ppm}\end{array}$ & $\begin{array}{l}1700 \\
\mathrm{ppm}\end{array}$ \\
\hline Pierre, SD & $\begin{array}{l}\mathrm{N} 0 \\
\mathrm{ppm}\end{array}$ & N/A & N/A & N/A & N/A & N/A & N/A & $\begin{array}{c}5.00 \\
\%\end{array}$ & $\begin{array}{l}\text { N0 } \\
\text { ppm }\end{array}$ & $\begin{array}{c}70 \\
\mathrm{ppm}\end{array}$ & $\begin{array}{l}500 \\
\mathrm{ppm}\end{array}$ \\
\hline Providence, RI & $\begin{array}{l}0.27 \\
\mathrm{ppm}\end{array}$ & N/A & $\begin{array}{c}47.20 \\
\% \\
\end{array}$ & $\begin{array}{c}12.50 \\
\%\end{array}$ & $\begin{array}{c}0.74 \\
\% \\
\end{array}$ & $\begin{array}{c}1.42 \\
\% \\
\end{array}$ & $\begin{array}{c}16.40 \\
\% \\
\end{array}$ & $\begin{array}{c}8.23 \\
\% \\
\end{array}$ & $\begin{array}{l}0.94 \\
\mathrm{ppm}\end{array}$ & $\begin{array}{r}286 \\
\mathrm{ppm}\end{array}$ & $\begin{array}{r}167 \\
\mathrm{ppm} \\
\end{array}$ \\
\hline Raleigh, NC & $\begin{array}{l}0.33 \\
\mathrm{ppm} \\
\end{array}$ & N/A & $\begin{array}{c}47.70 \\
\% \\
\end{array}$ & $\begin{array}{l}10 \\
\% \\
\end{array}$ & $\begin{array}{c}0.34 \\
\% \\
\end{array}$ & $\begin{array}{c}2.40 \\
\% \\
\end{array}$ & $\begin{array}{c}16.40 \\
\% \\
\end{array}$ & $\begin{array}{c}9.48 \\
\% \\
\end{array}$ & $\begin{array}{l}0.74 \\
\mathrm{ppm} \\
\end{array}$ & $\begin{array}{r}360 \\
\mathrm{ppm}\end{array}$ & $\begin{array}{c}71 \\
\mathrm{ppm}\end{array}$ \\
\hline Richmond, VA & $\begin{array}{l}\mathrm{L} 215 \\
\mathrm{ppm} \\
\end{array}$ & $\begin{array}{c}32.80 \\
\% \\
\end{array}$ & $\begin{array}{c}73.20 \\
\% \\
\end{array}$ & $\begin{array}{c}1.20 \\
\% \\
\end{array}$ & $\begin{array}{c}4.90 \\
\% \\
\end{array}$ & $\begin{array}{c}3.40 \\
\% \\
\end{array}$ & $\begin{array}{c}14.60 \\
\% \\
\end{array}$ & $\begin{array}{c}0.79 \\
\% \\
\end{array}$ & $\begin{array}{c}\text { L21.5 } \\
\text { ppm }\end{array}$ & $\begin{array}{l}173 \\
\mathrm{ppm} \\
\end{array}$ & $\begin{array}{r}537 \\
\text { ppm } \\
\end{array}$ \\
\hline Salem, OR & N/A & $\begin{array}{c}\text { G10 } \\
\% \\
\end{array}$ & $\begin{array}{c}54.80 \\
\% \\
\end{array}$ & $\begin{array}{c}6.90 \\
\% \\
\end{array}$ & $\begin{array}{c}0.82 \\
\% \\
\end{array}$ & $\begin{array}{c}3.50 \\
\% \\
\end{array}$ & $\begin{array}{c}17.90 \\
\% \\
\end{array}$ & $\begin{array}{c}7.00 \\
\% \\
\end{array}$ & $\begin{array}{c}\text { N0 } \\
\text { ppm }\end{array}$ & $\begin{array}{l}200 \\
\mathrm{ppm}\end{array}$ & $\begin{array}{r}300 \\
\mathrm{ppm} \\
\end{array}$ \\
\hline Salt Lake City, UT & $\begin{array}{c}\text { N0 } \\
\text { ppm }\end{array}$ & N/A & $\mathrm{N} / \mathrm{A}$ & N/A & N/A & N/A & N/A & $\begin{array}{c}0.15 \\
\% \\
\end{array}$ & $\begin{array}{c}\text { N0 } \\
\text { ppm }\end{array}$ & $\begin{array}{r}200 \\
\mathrm{ppm}\end{array}$ & $\begin{array}{c}50 \\
\mathrm{ppm}\end{array}$ \\
\hline San Francisco, CA & $\begin{array}{c}\mathrm{N} 0 \\
\mathrm{ppm}\end{array}$ & $\begin{array}{c}\text { G10 } \\
\% \\
\end{array}$ & $\begin{array}{c}71.60 \\
\% \\
\end{array}$ & $\begin{array}{c}1.50 \\
\% \\
\end{array}$ & $\begin{array}{c}2.30 \\
\% \\
\end{array}$ & $\begin{array}{c}3 \\
\% \\
\end{array}$ & $\begin{array}{c}13.90 \\
\% \\
\end{array}$ & $\begin{array}{c}2 \\
\% \\
\end{array}$ & $\begin{array}{c}\text { N0 } \\
\text { ppm }\end{array}$ & $\begin{array}{l}200 \\
\mathrm{ppm}\end{array}$ & $\begin{array}{l}500 \\
\mathrm{ppm}\end{array}$ \\
\hline Santa Fe, NM & $\begin{array}{l}17.2 \\
\mathrm{ppm}\end{array}$ & N/A & $\begin{array}{c}49.90 \\
\% \\
\end{array}$ & $\begin{array}{c}10.10 \\
\%\end{array}$ & $\begin{array}{c}0.58 \\
\% \\
\end{array}$ & $\begin{array}{c}2.40 \\
\% \\
\end{array}$ & $\begin{array}{c}13.30 \\
\% \\
\end{array}$ & $\begin{array}{c}0.90 \\
\% \\
\end{array}$ & $\begin{array}{l}43.2 \\
\mathrm{ppm}\end{array}$ & $\begin{array}{l}\text { L110 } \\
\text { ppm }\end{array}$ & $\begin{array}{l}102 \\
\mathrm{ppm}\end{array}$ \\
\hline Seattle, WA & N/A & N/A & $\begin{array}{c}59.30 \\
\% \\
\end{array}$ & $\begin{array}{c}5.70 \\
\% \\
\end{array}$ & $\begin{array}{c}1.01 \\
\% \\
\end{array}$ & $\begin{array}{c}4.81 \\
\% \\
\end{array}$ & $\begin{array}{c}17.90 \\
\% \\
\end{array}$ & N/A & N/A & N/A & N/A \\
\hline Springfield, IL & $\begin{array}{l}\mathrm{L} 100 \\
\mathrm{ppm}\end{array}$ & N/A & N/A & N/A & N/A & N/A & N/A & $\begin{array}{c}3.70 \\
\% \\
\end{array}$ & $\begin{array}{c}9 \\
\mathrm{ppm} \\
\end{array}$ & $\begin{array}{l}120 \\
\mathrm{ppm} \\
\end{array}$ & $\begin{array}{r}270 \\
\mathrm{ppm} \\
\end{array}$ \\
\hline Topeka, KS & $\begin{array}{c}\mathrm{N} 0 \\
\mathrm{ppm}\end{array}$ & $\begin{array}{c}1.50 \\
\%\end{array}$ & N/A & N/A & N/A & N/A & N/A & $\begin{array}{c}1.00 \\
\%\end{array}$ & $\begin{array}{c}\text { N0 } \\
\text { ppm }\end{array}$ & $\begin{array}{r}700 \\
\mathrm{ppm}\end{array}$ & $\begin{array}{c}30 \\
\mathrm{ppm}\end{array}$ \\
\hline Trenton, NJ & $\begin{array}{l}0.82 \\
\mathrm{ppm} \\
\end{array}$ & N/A & N/A & N/A & N/A & N/A & N/A & $\begin{array}{c}9.02 \\
\% \\
\end{array}$ & $\begin{array}{r}3.6 \\
\mathrm{ppm} \\
\end{array}$ & $\begin{array}{r}257 \\
\mathrm{ppm} \\
\end{array}$ & $\begin{array}{r}235 \\
\mathrm{ppm} \\
\end{array}$ \\
\hline Washington D.C. & $\begin{array}{c}\mathrm{N} 0 \\
\mathrm{ppm}\end{array}$ & $\begin{array}{c}\text { G10 } \\
\% \\
\end{array}$ & $\begin{array}{c}55.10 \\
\% \\
\end{array}$ & $\begin{array}{c}0.08 \\
\% \\
\end{array}$ & $\begin{array}{c}0.11 \\
\% \\
\end{array}$ & $\begin{array}{c}0.08 \\
\% \\
\end{array}$ & $\begin{array}{c}4.20 \\
\% \\
\end{array}$ & $\begin{array}{c}7 \\
\% \\
\end{array}$ & $\begin{array}{c}\text { N0 } \\
\text { ppm }\end{array}$ & $\begin{array}{c}30 \\
\mathrm{ppm}\end{array}$ & $\begin{array}{l}3000 \\
\mathrm{ppm}\end{array}$ \\
\hline
\end{tabular}




\section{DATA FROM BUILDING MATERIALS}

The following data set (Table 4) lists activity concentrations for Uranium, Thorium, Potassium, and Radium in various building materials. This data is important for analyzing element concentrations of post-detonation debris from an urban area. Using this information, scientists can determine the amount of these elements in a sample that came from the existing building materials.

4. Activity Concentrations in Building Materials

\begin{tabular}{|l|l|l|l|l|}
\hline Building Material & 232 Th Bq/kg & 226Ra Bq/kg & 40K Bq/kg & 238U Bq/kg \\
\hline Aerated clay & 20 & 30 & 570 & N/A \\
\hline Aerated concrete w/shale & 30 & 25 & 1180 & N/A \\
\hline Aerated schist & 50 & 60 & 860 & N/A \\
\hline Brick & 46.88 & 21.7 & 406.6 & 73 \\
\hline Brick w/fly ash & 105 & 135 & 140 & N/A \\
\hline Cement & 55.2 & 25.8 & 299.8 & 98 \\
\hline Concrete w/coal fly ash & 50 & 10 & 450 & N/A \\
\hline Concrete w/ copper slag & 65 & 35 & 970 & N/A \\
\hline Copper slag & 230 & 40 & 730 & N/A \\
\hline Dholpur stone & 33 & N/A & 1425 & 49 \\
\hline Foundry slag & 75 & 60 & 120 & N/A \\
\hline Granite & 98.8 & 49.4 & 1456.1 & 82 \\
\hline Hematite aggregate & 26 & N/A & 288 & 68 \\
\hline Lignite fly ash & 55 & 25 & 310 & N/A \\
\hline Marble & BDL & N/A & 230 & 29 \\
\hline Mosaic tile & 76 & N/A & 353 & 85 \\
\hline Ordinary aggregate & 67 & N/A & 1009 & 60 \\
\hline Radiation shielding bricks & 109 & N/A & 1030 & 89 \\
\hline Rocks & 11.7 & 6.6 & 217.6 & N/A \\
\hline
\end{tabular}




\begin{tabular}{|l|l|l|l|l|}
\hline Sand & 38.8 & 11.4 & 743.3 & 40 \\
\hline Sedam cement & 29.4 & 25.8 & 259.6 & N/A \\
\hline Slag stone & 45 & 60 & 90 & N/A \\
\hline Soil & 20 & N/A & 200 & 30 \\
\hline Stone w/ coal fly ash & 85 & 65 & 910 & N/A \\
\hline
\end{tabular}

$\mathrm{BDL}=$ below detectible level

\section{USING PYTHON}

The data has been formatted so that it can be accessed through a python program. Python is the program of choice as it is simple and has wide uses. A brief description of the program and its interface follows.

This program made use of the CSV module in Python. This module allows programmers to access data stored in CSV files and run that data through a program. The first step in writing this program is to convert the desired data source into a CSV file. It is also important to ensure that the data file is saved in the same location as the program itself.

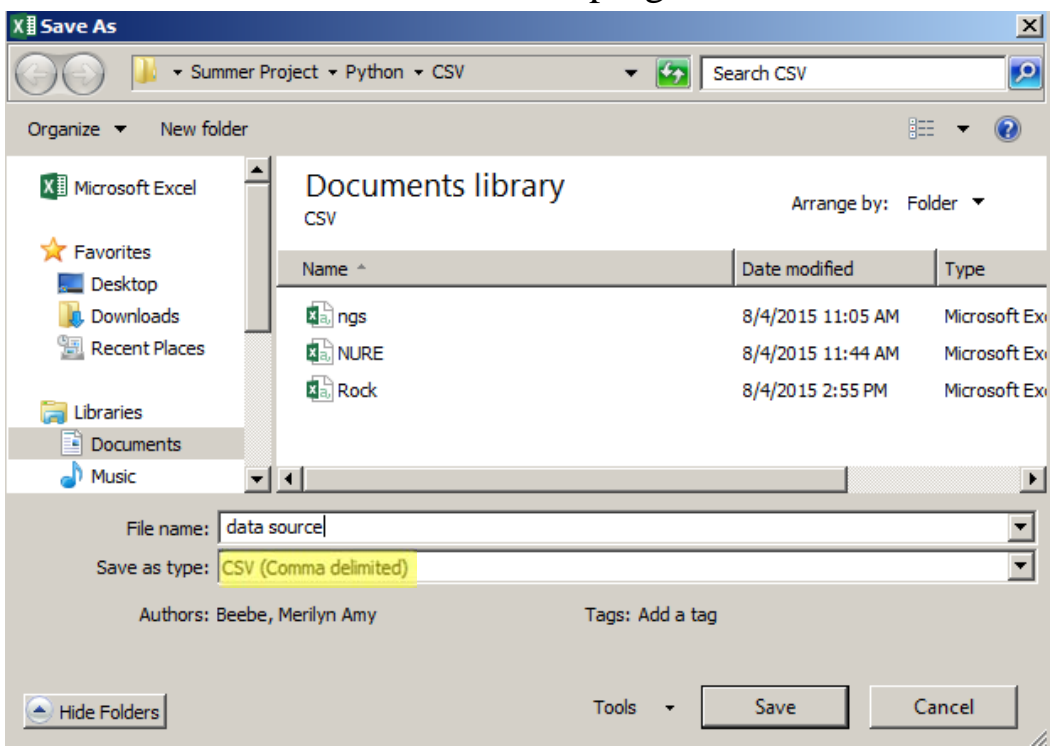

After setting up the CSV module in the program, the data is assigned to lists. The first column of the excel file lists the cities where data was found. The program stores each of these cities into a list. The same is done for the columns containing the data. Each city is assigned an index within the city list.

Index 0

Index 1

Index 2

And so on ...

[", 'ALBUQUERQUE', 'ANNAPOLIS', 'ATLANTA', 'AUGUSTA', 'BATON ROUGE', 'BISMARCK', 'BOISE', 'CARSON CITY', 'CHARLESTON', 'CHEYENNE' , 'CHICAGO', 'COLUMBUS', 'CONCORD', 'DALLAS', 'DES MOINES', 'DETROIT', 'DOVER', 'EL PASO', 'FORT WORTH', 'FRANKFORT', 'HARTFORD', 'H OUSTON', 'INDIANAPOLIS', 'JACKSON', 'JACKSONVILLE', 'JEFFERSON CITY', 'JUNEAU', 'LINCOLN', 'LITTLE ROCK', 'LOS ANGELES', 'MONTPELI ER', 'NASHVILLE', 'OKLAHOMA CITY', 'PHOENIX', 'PIERRE', 'RALEIGH', 'RICHMOND', 'SACRAMENTO', 'SALEM', 'SALT LAKE CITY', 'SAN ANTONIO 
This index corresponds to the location data from that city in the data list.

Index $0 \quad$ Index $1 \quad$ Index $2 \quad$ And so on ...

['th', '11 PPM', '8 PPM', '33 PPM', '13 PPM', '9 PPM', '14 PPM', '19 PPM', '< 6 PPMt, '14 PPM', '14 PPM', '6 PPM', '11 PPM', '26 PPM', '10 PPM', '10 PPM ', '< 6 PPM ', '11 PPM', '8 PPM', '10 PPM ', '9 PPM', '15 PPM', '< 6 PPM ', '< 6 PPM ', '12 PPM', '8 PPM', '10 PPM', '14 PPM', '10 PPM', '12 PPM', '15 PPM' , '10 PPM', '12 PPM', '8 PPM', '12 PPM', '8 PPM', '14 PPM', '13 PPM', '6 PPM', '12 PPM', '11 PPM', '<6 PPM', '9 PPM', '20 PPM', '12 PPM', '10 PPM', '9 P $\mathrm{PM}$ ']

indexes and produces the data.

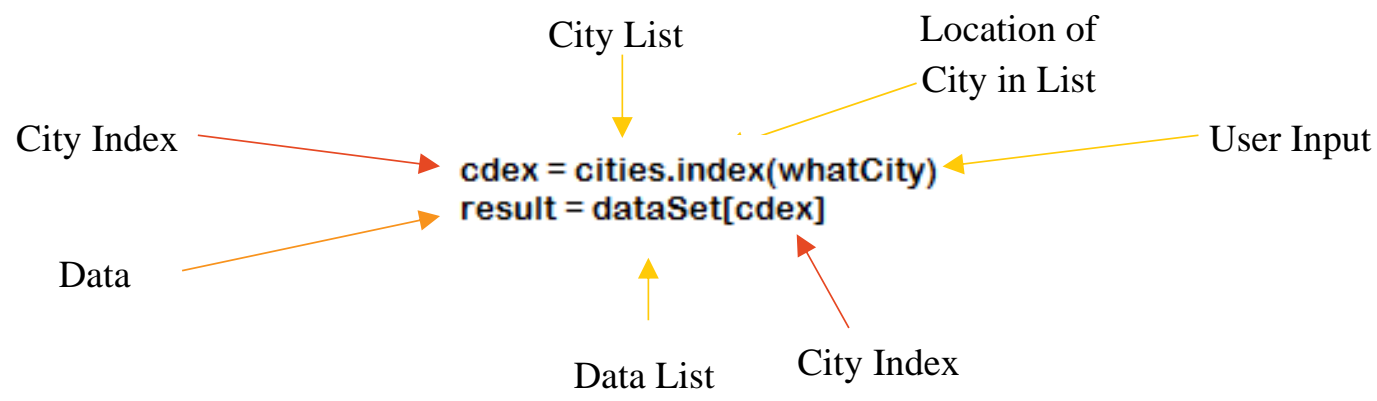

The result, depending on the user inputs, looks something like this:

The element concentration of THORIUM in TOPEKA is 9 PPM

Finally, the program contains a repeat sequence that allows the user to conduct multiple searches before exiting the program.

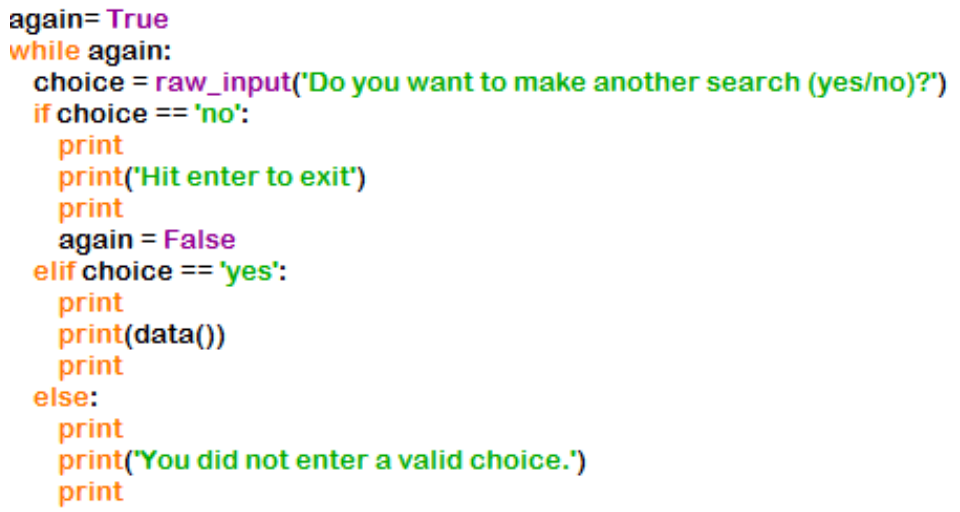

The whole program consists of approximately 100 lines of code. This program, not including the code written in the CSV module, is very short, and this method for storing and accessing the data was the simplest and easiest to modify. Wherever possible, variables are used 
so that any updates made effect the entire program. In addition, the lines of code that read 'print,' do not have a real purpose in the program other than to make the output easy to read. The use of the CSV made it possible to catalog and access the data using Excel rather than logging all of the data in the program itself.

\section{SOURCES}

"Albert Einstein." Bio. A\&E Television Networks, 2015. Web. 21 July 2015. http://www.biography.com/people/albert-einstein-9285408\#final-years

"Atoms for Peace." Atoms for Peace. IAEA, n.d. Web. 20 July 2015. https://www.iaea.org/about

"Enrico Fermi - Biographical". Nobelprize.org. Nobel Media AB 2014. Web. 14 Jul 2015. http://www.nobelprize.org/nobel_prizes/physics/laureates/1938/fermi-bio.html

"Henri Becquerel - Biographical". Nobelprize.org. Nobel Media AB 2014. Web. 14 Jul 2015. http://www.nobelprize.org/nobel_prizes/physics/laureates/1903/becquerel-bio.html

"Marie Curie." Bio. A\&E Television Networks, 2015. Web. 14 July 2015. http://www.biography.com/people/marie-curie-9263538\#video-gallery

"Nuclear Power Plants." Nuclear Power Plants. Ready, 02 Nov. 2013. Web. 20 July 2015. http://www.ready.gov/nuclear-power-plants

Szaflarski, Diane, Robert Dean, and Melaine Dean. "Nuclear Chemistry." Nuclear Chemistry. $\begin{array}{lllll}\text { DOE, } & \text { n.d. } & \text { Web. } & 20 & \text { July }\end{array}$ http://people.chem.duke.edu/ jds/cruise_chem/nuclear/exposure.html

"The Advancement of Nuclear Chemistry." Timetoast. N.p., n.d. Web. 14 July 2015. http://www.timetoast.com/timelines/the-advancement-of-nuclear-chemistry

"The Gold Foil Experiment." The Gold Foil Experiment. N.p., n.d. Web. 14 July 2015. http://myweb.usf.edu/ mhight/goldfoil.html

"The Manhattan Project." Ushistory.org. Independence Hall Association, n.d. Web. 14 July 2015. http://www.ushistory.org/us/51f.asp

"Wilhelm Conrad Röntgen - Biographical". Nobelprize.org. Nobel Media AB 2014. Web. 14 Jul 2015. http://www.nobelprize.org/nobel_prizes/physics/laureates/1901/rontgen-bio.html 
"Your Geography Selections." American FactFinder. U.S Census Bureau, Population Division, $\begin{array}{lllll}\text { May } & 2015 . & \text { Web. } & 18 & \text { June }\end{array}$ http://factfinder.census.gov/faces/tableservices/jsf/pages/productview.xhtml?src=bkmk

National Geochemical Survey Data

"National Geochemical Survey Database." National Geochemical Survey Database. USGS, 03

$\begin{array}{llllll}\text { Sept. } & 2014 . & \text { Web. } & 20 & \text { July }\end{array}$

http://mrdata.usgs.gov/geochemistry/ngs.html

Los Angeles: http://mrdata.usgs.gov/ngs/show-ngs.php?labno=C-195964

Chicago: http://mrdata.usgs.gov/ngs/show-ngs.php?labno=D-305577

Houston: http://mrdata.usgs.gov/ngs/show-ngs.php?labno=C-155090

Phoenix: http://mrdata.usgs.gov/ngs/show-ngs.php?labno=C-180488

San Antonio: http://mrdata.usgs.gov/ngs/show-ngs.php?labno=C-149739

Dallas: http://mrdata.usgs.gov/ngs/show-ngs.php?labno=C-155171

San Jose: http://mrdata.usgs.gov/ngs/show-ngs.php?labno=C-196079

Jacksonville: http://mrdata.usgs.gov/ngs/show-ngs.php?labno=C-112206

Indianapolis: http://mrdata.usgs.gov/ngs/show-ngs.php?labno=C-261788

Columbus: http://mrdata.usgs.gov/ngs/show-ngs.php?labno=C-258007

Fort Worth: http://mrdata.usgs.gov/ngs/show-ngs.php?labno=C-161767

Detroit: http://mrdata.usgs.gov/ngs/show-ngs.php?labno=C-194538

El Paso: http://mrdata.usgs.gov/ngs/show-ngs.php?labno=D-558899

Albuquerque: http://mrdata.usgs.gov/ngs/show-ngs.php?labno=C-159015

Santa Fe: http://mrdata.usgs.gov/ngs/show-ngs.php?labno=C-165150

Juneau: http://mrdata.usgs.gov/ngs/show-ngs.php?labno=C-178683

Little Rock: http://mrdata.usgs.gov/ngs/show-ngs.php?labno=C-127241

Sacramento: http://mrdata.usgs.gov/ngs/show-ngs.php?labno=C-165147 
Hartford: http://mrdata.usgs.gov/ngs/show-ngs.php?labno=C-145708

Dover: http://mrdata.usgs.gov/ngs/show-ngs.php?labno=C-211251

Tallahassee: http://mrdata.usgs.gov/ngs/show-ngs.php?labno=C-123498

Atlanta: http://mrdata.usgs.gov/ngs/show-ngs.php?labno=C-211738

Boise: http://mrdata.usgs.gov/ngs/show-ngs.php?labno=C-168047

Springfield: http://mrdata.usgs.gov/ngs/show-ngs.php?labno=C-224605

Des Moines: http://mrdata.usgs.gov/ngs/show-ngs.php?labno=C-236357

Topeka: http://mrdata.usgs.gov/ngs/show-ngs.php?labno=C-279425

Frankfort: http://mrdata.usgs.gov/ngs/show-ngs.php?labno=C-256099

Baton Rouge: http://mrdata.usgs.gov/ngs/show-ngs.php?labno=C-183789

Augusta: http://mrdata.usgs.gov/ngs/show-ngs.php?labno=C-148047

Annapolis: http://mrdata.usgs.gov/ngs/show-ngs.php?labno=C-117040

Jackson: http://mrdata.usgs.gov/ngs/show-ngs.php?labno=C-102902

Jefferson City: http://mrdata.usgs.gov/ngs/show-ngs.php?labno=C-262073

Lincoln: http://mrdata.usgs.gov/ngs/show-ngs.php?labno=C-150069

Carson City: http://mrdata.usgs.gov/ngs/show-ngs.php?labno=C-164137

Concord: http://mrdata.usgs.gov/ngs/show-ngs.php?labno=C-145806

Raleigh: http://mrdata.usgs.gov/ngs/show-ngs.php?labno=C-210806

Bismarck: http://mrdata.usgs.gov/ngs/show-ngs.php?labno=C-255370

Oklahoma City: http://mrdata.usgs.gov/ngs/show-ngs.php?labno=C-152447

Salem: http://mrdata.usgs.gov/ngs/show-ngs.php?labno=C-262410

Pierre: http://mrdata.usgs.gov/ngs/show-ngs.php?labno=C-297871

Nashville: http://mrdata.usgs.gov/ngs/show-ngs.php?labno=C-279056

Salt Lake City: http://mrdata.usgs.gov/ngs/show-ngs.php?labno=C-144767

Montpelier: http://mrdata.usgs.gov/ngs/show-ngs.php?labno=C-148423 
Richmond: http://mrdata.usgs.gov/ngs/show-ngs.php?labno=C-211701

Charleston: http://mrdata.usgs.gov/ngs/show-ngs.php?labno=C-143526

Cheyenne: http://mrdata.usgs.gov/ngs/show-ngs.php?labno=C-152103

$\underline{\text { National Uranium Resource Evaluation Data }}$

"Geochemistry of Stream Sediments from NURE-HSSR." Geochemistry of Stream Sediments from NURE-HSSR. USGS, 03 Sept. 2014. Web. 20 July 2015. http://mrdata.usgs.gov/geochemistry/nuresed.html

Phoenix: http://mrdata.usgs.gov/nuresed/show-nuresed.php?rec_no=5206016

San Antonio: http://mrdata.usgs.gov/nuresed/show-nuresed.php?rec_no=5237110

Dallas: http://mrdata.usgs.gov/nuresed/show-nuresed.php?rec_no=5242174

Austin: http://mrdata.usgs.gov/nuresed/show-nuresed.php?rec_no=5247929

Fort Worth: http://mrdata.usgs.gov/nuresed/show-nuresed.php?rec_no=5242127

Montgomery: http://mrdata.usgs.gov/nuresed/show-nuresed.php?rec_no=5268422

Juneau: http://mrdata.usgs.gov/nuresed/show-nuresed.php?rec_no=7020084

Little Rock: http://mrdata.usgs.gov/nuresed/show-nuresed.php?rec_no=5252112

Sacramento: http://mrdata.usgs.gov/nuresed/show-nuresed.php?rec_no=5190291

Denver: http://mrdata.usgs.gov/nuresed/show-nuresed.php?rec_no=5078759

Hartford: http://mrdata.usgs.gov/nuresed/show-nuresed.php?rec_no=5329804

Dover: http://mrdata.usgs.gov/nuresed/show-nuresed.php?rec_no=5313307

Atlanta: http://mrdata.usgs.gov/nuresed/show-nuresed.php?rec_no=5276853

Boise: http://mrdata.usgs.gov/nuresed/show-nuresed.php?rec_no=5180136

Augusta: http://mrdata.usgs.gov/nuresed/show-nuresed.php?rec_no=5275377

Annapolis: http://mrdata.usgs.gov/nuresed/show-nuresed.php?rec_no=5311258

Helena: http://mrdata.usgs.gov/nuresed/show-nuresed.php?rec_no=5023390 
Lincoln: http://mrdata.usgs.gov/nuresed/show-nuresed.php?rec_no=5214684

Carson City: http://mrdata.usgs.gov/nuresed/show-nuresed.php?rec_no=5154056

Concord: http://mrdata.usgs.gov/nuresed/show-nuresed.php?rec_no=5326626

Trenton: http://mrdata.usgs.gov/nuresed/show-nuresed.php?rec_no=5315016

Santa Fe: http://mrdata.usgs.gov/nuresed/show-nuresed.php?rec_no=5107835

Albany: http://mrdata.usgs.gov/nuresed/show-nuresed.php?rec_no=5317008

Raleigh: http://mrdata.usgs.gov/nuresed/show-nuresed.php?rec_no=5287290

Oklahoma City: http://mrdata.usgs.gov/nuresed/show-nuresed.php?rec_no=5242991

Harrisburg: http://mrdata.usgs.gov/nuresed/show-nuresed.php?rec_no=5314096

Providence: http://mrdata.usgs.gov/nuresed/show-nuresed.php?rec_no=5327748

Columbia; http://mrdata.usgs.gov/nuresed/show-nuresed.php?rec_no=5294723

Salt Lake City: http://mrdata.usgs.gov/nuresed/show-nuresed.php?rec_no=5106472

Montpelier: http://mrdata.usgs.gov/nuresed/show-nuresed.php?rec_no=5322754

Richmond; http://mrdata.usgs.gov/nuresed/show-nuresed.php?rec_no=5304343

Cheyenne: http://mrdata.usgs.gov/nuresed/show-nuresed.php?rec_no=5050659

\section{$\underline{\text { Rock Data }}$}

"National Geochemical Database: Rock." National Geochemical Database: Rock. USGS, 03

$\begin{array}{llllll}\text { Sept. } & 2014 . & \text { Web. } & 20 & \text { July } & 2015 .\end{array}$

http://mrdata.usgs.gov/geochemistry/ngdbrock.html

New York: http://mrdata.usgs.gov/ngdb/rock/show-ngdbrock.php?lab_id=W177284

Los Angeles: http://mrdata.usgs.gov/ngdb/rock/show-ngdbrock.php?lab_id=D219632

El Paso: http://mrdata.usgs.gov/ngdb/rock/show-ngdbrock.php?lab_id=D154981

Albuquerque: http://mrdata.usgs.gov/ngdb/rock/show-ngdbrock.php?lab_id=D178443 
http://mrdata.usgs.gov/ngdb/rock/show-ngdbrock.php?lab_id=D163055

Phoenix: http://mrdata.usgs.gov/ngdb/rock/show-ngdbrock.php?lab_id=M201019

Seattle: http://mrdata.usgs.gov/ngdb/rock/show-ngdbrock.php?lab_id=D563851

Charlotte: http://mrdata.usgs.gov/ngdb/rock/show-ngdbrock.php?lab_id=W207654

Washington D.C.: http://mrdata.usgs.gov/ngdb/rock/show-ngdbrock.php?lab_id=W170069

Baltimore: http://mrdata.usgs.gov/ngdb/rock/show-ngdbrock.php?lab_id=D539118

Austin: http://mrdata.usgs.gov/ngdb/rock/show-ngdbrock.php?lab_id=D183421

San Francisco: http://mrdata.usgs.gov/ngdb/rock/show-ngdbrock.php?lab_id=M120466

Columbus: http://mrdata.usgs.gov/ngdb/rock/show-ngdbrock.php?lab_id=D124008

Juneau: http://mrdata.usgs.gov/ngdbrock/show-ngdbrock.php?lab_id=M174567

Little Rock: http://mrdata.usgs.gov/ngdb/rock/show-ngdbrock.php?lab_id=D116559

Hartford: http://mrdata.usgs.gov/ngdb/rock/show-ngdbrock.php?lab_id=W237385

Atlanta: http://mrdata.usgs.gov/ngdb/rock/show-ngdbrock.php?lab_id=W215182

Boise: http://mrdata.usgs.gov/ngdb/rock/show-ngdbrock.php?lab_id=D345878

Honolulu: http://mrdata.usgs.gov/ngdbrock/show-ngdbrock.php?lab_id=M126256

Springfield: http://mrdata.usgs.gov/ngdb/rock/show-ngdbrock.php?lab_id=D517473

Topeka: http://mrdata.usgs.gov/ngdb/rock/show-ngdbrock.php?lab_id=D148145

Frankfort: http://mrdata.usgs.gov/ngdb/rock/show-ngdbrock.php?lab_id=W182677

Augusta: http://mrdata.usgs.gov/ngdb/rock/show-ngdbrock.php?lab_id=W172068

Jefferson City: http://mrdata.usgs.gov/ngdb/rock/show-ngdbrock.php?lab_id=D150445

Carson City: http://mrdata.usgs.gov/ngdb/rock/show-ngdbrock.php?lab_id=D236160

Concord: http://mrdata.usgs.gov/ngdb/rock/show-ngdbrock.php?lab_id=W196609

Trenton: http://mrdata.usgs.gov/ngdb/rock/show-ngdbrock.php?lab_id=W243095

Santa Fe: http://mrdata.usgs.gov/ngdb/rock/show-ngdbrock.php?lab_id=W193230 http://mrdata.usgs.gov/ngdb/rock/show-ngdbrock.php?lab_id=D191472 
Raleigh: http://mrdata.usgs.gov/ngdb/rock/show-ngdbrock.php?lab_id=W231948

Oklahoma City: http://mrdata.usgs.gov/ngdb/rock/show-ngdbrock.php?lab_id=D290526

Salem: http://mrdata.usgs.gov/ngdb/rock/show-ngdbrock.php?lab_id= D290526

Harrisburg: http://mrdata.usgs.gov/ngdb/rock/show-ngdbrock.php?lab_id=W243867

Providence: http://mrdata.usgs.gov/ngdb/rock/show-ngdbrock.php?lab_id=W251884

Columbia: http://mrdata.usgs.gov/ngdb/rock/show-ngdbrock.php?lab_id=W179520

Pierre: http://mrdata.usgs.gov/ngdb/rock/show-ngdbrock.php?lab_id=D155231

Nashville: http://mrdata.usgs.gov/ngdb/rock/show-ngdbrock.php?lab_id=D236689

Salt Lake City: http://mrdata.usgs.gov/ngdb/rock/show-ngdbrock.php?lab_id=D163680

Montpelier: http://mrdata.usgs.gov/ngdb/rock/show-ngdbrock.php?lab_id=D577702

Richmond: http://mrdata.usgs.gov/ngdb/rock/show-ngdbrock.php?lab_id=W182140

Charleston: http://mrdata.usgs.gov/ngdb/rock/show-ngdbrock.php?lab_id=W220493

Cheyenne: http://mrdata.usgs.gov/ngdb/rock/show-ngdbrock.php?lab_id=D122240

\section{Building Materials}

Keller, G., B. Hoffmann, and T. Feigenspan. "Radon Permeability and Radon Exhalation of Building Materials." Science Direct. Elsevier, 14 May 2001. Web. 20 July 2015.

Kerur, Basavaraj R., Tanakanti Rajeshwari, Rajesh Siddanna, and Anil S. Kumar. "2013 Acta Geophysica Full." ResearchGate. Versits, n.d. Web. 20 July 2015.

Sonkawade, R. G., K. Kant, S. Muralithar, R. C. Ramola, and R. Kumar. "Natural Radioactivity in Common Building Construction and Radiation Shielding Materials." Science Direct. Elsevier, Mar. 2008. Web. 20 July 2015. 